Service social

\title{
La mesure et l'amélioration de la productivité dans les services sociaux : des choix difficiles
}

\section{Jean Harvey}

Volume 36, numéro 1, 1987

L’informatique dans les services sociaux

URI : https://id.erudit.org/iderudit/706345ar

DOI : https://doi.org/10.7202/706345ar

Aller au sommaire du numéro

\section{Éditeur(s)}

École de service social de l'Université Laval

ISSN

1708-1734 (numérique)

Découvrir la revue

Citer ce document

Harvey, J. (1987). La mesure et l'amélioration de la productivité dans les services sociaux : des choix difficiles. Service social, 36(1), 129-147. https://doi.org/10.7202/706345ar

\section{Résumé de l'article}

La mesure de la productivité est difficile dans les services professionnels en général et dans les services sociaux en particulier. Puisqu'il est impossible d'obtenir des indices de productivité inattaquables, plusieurs se résignent à gérer sans indices. Une autre avenue consiste à mettre sur pied des programmes d'amélioration de la productivité basés sur des mesures imparfaites et/ou incomplètes, mesurant davantage l'efficience que l'efficacité. Ceux qui les utilisent doivent toutefois être conscients des limites de ces indices et soucieux de les améliorer constamment.

Différentes approches sont présentées : le décompte des activités des praticiens, les matrices multicritères et le décompte des cas traités ; leurs avantages et inconvénients respectifs sont discutés. Après une présentation de certains aspects critiques à considérer lors de la mise en place de tels indices soit la dynamique de comparaison à instaurer et le développement organisationnel nécessaire - quatre expériences québécoises récentes ou en cours sont présentées sommairement. 


\title{
COMMENTAIRES ET DOCUMENTS
}

HARVEY, Jean, professeur au Département des sciences administratives de I'Université du Québec à Montréal.

\section{La mesure et l'amélioration de la productivité dans les services sociaux : des choix difficiles}

\author{
Jean Harvey
}

L'augmentation de la productivité dans le secteur des services en général a toujours posé des défis de taille aux gestionnaires (Britney, Johnson, Legentil et Walsh, 1982). Mesurer la productivité est un prérequis à tout effort systématique et concerté pour l'améliorer. (Kendrick, 1984).

La mesure s'avère particulièrement difficile dans les services professionnels, compte tenu de leur intangibilité. Les résultats de ces services ne se font souvent ressentir qu'à long terme. De plus, il s'avère souvent difficile de s'assurer que les services professionnels rendus en sont la cause majeure. Ces problèmes de mesure sont tels qu'il est facile de s'y embourber :

"Malheureusement, de nombreuses compagnies relèguent au second rang la question de la mesure, parce qu'on ne peut s'entendre sur des questions comme où mesurer, quels facteurs considérer, et comment intégrer cela à une analyse financière. " (Kendrick, 1984 : 119.) 


\section{Les enjeux}

Le Ministère de la santé et des services sociaux du Québec (M.S.S.S.) a procédé récemment à une réaffectation massive de ressources des Centres de service sociaux (C.S.S.) vers les Centres locaux de services communautaires (C.L.S.C.). Pour déterminer la quantité de ressources à retrancher de chaque C.S.S., le Ministère a dû se baser sur la "production " - c'est-à-dire le nombre de dossiers traités - et les charges de travail relatives dans chaque C.S.S. Pour obtenir les données requises, le Ministère s'est tout normalement tourné vers les sources officielles: les rapports annuels soumis par les C.S.S. (D.G.F.-1, D.G.F.-2). Or ces données n'avaient pas atteint un niveau de fiabilité et d'uniformité suffisant pour se prêter à un tel usage. Elles ne reflétaient donc pas les niveaux d'activités et les charges de travail réels dans les différents C.S.S.. Les conséquences de la réaffectation de ressources à laquelle le Ministère a procédé, compte tenu principalement de son envergure, peuvent donc pénaliser indûment certaines populations et certains professionnels.

L'absence d'indices de productivité acceptés par tous les intervenants peut aussi causer des problèmes plus près de la base. Ainsi, deux services cliniques, œuvrant dans le même programme, dans un même C.S.S., peuvent faire face à des charges de travail différentes; de plus, les pratiques administratives et professionnelles peuvent varier entre eux. Pourtant, si aucun indice valable de gestion n'attire l'attention du directeur de ce programme sur ces écarts, comment peut-on s'y prendre pour démontrer l'inéquité de la situation et pour la corriger?

Bien qu'intervenant à des plans différents, ces deux situations sont le reflet d'une même problématique : l'absence virtuelle de mesure même approximative - de la productivité dans les services sociaux, au Québec et ailleurs, et ses conséquences sur la gestion et l'amélioration de la productivité.

Cette problématique présente toutefois des facettes différentes selon les intervenants et leurs optiques et objectifs particuliers. Pour les fins du présent article, on peut regrouper les intervenants en cinq grandes catégories : le M.S.S.S., la direction générale et la direction des services professionnels (D.S.P.) du C.S.S., les cadres de première ligne ou chefs de service et les praticiens (ce terme désigne ici tous les intervenants sociaux : travailleurs sociaux, criminologues, psychologues, etc.) Le tableau 1 résume succinctement les principales décisions de chaque intervenant pour lesquelles des indices de productivité sont requis. 
TABLEAU 1

\section{Besoins des principaux intervenants face aux indices de productivité}

\begin{tabular}{|c|c|}
\hline Intervenant & Décisions pour lesquelles des indices sont requis \\
\hline M.S.S.S. & $\begin{array}{l}\text { Détermination des ressources totales requises pour le Québec } \\
\text { dans les services sociaux } \\
\text { Affectation des ressources entre les régions et entre les programmes }\end{array}$ \\
\hline $\begin{array}{l}\text { Direction } \\
\text { générale }\end{array}$ & $\begin{array}{l}\text { Établissement des priorités de service } \\
\text { Détermination des politiques de service } \\
\text { Affectation interne des ressources entre les sous-régions et les } \\
\text { programmes }\end{array}$ \\
\hline D.S.P. & $\begin{array}{l}\text { Établissement des politiques professionnelles } \\
\text { Formulation des programmes } \\
\text { Assurance de la qualité } \\
\text { Arbitrage qualité/quantité }\end{array}$ \\
\hline $\begin{array}{l}\text { Cadre de } \\
\text { première } \\
\text { ligne }\end{array}$ & $\begin{array}{l}\text { Amélioration de la productivité } \\
\text { Établissement des priorités opérationnelles } \\
\text { Gestion de la charge de travail } \\
\text { Gestion de la liste d'attente }\end{array}$ \\
\hline Praticien & $\begin{array}{l}\text { Amélioration de leur efficacité } \\
\text { Gestion de leur charge de travail } \\
\text { Connaissance de leur champ d'intervention, de même que des } \\
\text { attentes du C.S.S. }\end{array}$ \\
\hline
\end{tabular}

\section{Principales approches possibles}

Il y a deux grandes approches possibles pour obtenir des indices de productivité dans les services sociaux: la mesure des intrants et la mesure des extrants.

La mesure des intrants consiste à élaborer des indices pour recenser, de façon plus ou moins détaillée, les activités des praticiens. Sime et Farber (1982) ont utilisé une telle approche, basée sur l'utilisation d'un journal quotidien d'activités. Ils ont défini au-delà de cinquante activités qu'un praticien est susceptible de réaliser au cours d'une journée, 
regroupées sous quatre grandes rubriques : services directs aux patients (par exemple une entrevue de psychothérapie), services pour le compte des patients (correspondance, rapports d'évaluation, etc.), services aux institutions et autres partenaires, administration du service. Pour tenir compte du fait que certaines activités sont plus exigeantes que d'autres, ils ont assorti chaque activité d'un indice de difficulté qu'ils ont estimé avec la collaboration des praticiens. Ils ont ainsi obtenu un indice de performance basé sur l'équivalence-heure $(\mathrm{EH}):^{1}$

\section{$\mathrm{EH}=$ heures consacrées à l'activité $\times$ indice de difficulté}

Des expériences furent réalisées dans le cadre de services sociaux hospitaliers et les participants trouvèrent utiles les données résultant de cette approche.

Toutefois, puisque cet indice n'est nullement basé sur les extrants ou les résultats obtenus, il est tout à fait possible qu'une augmentation de l'indice $\mathrm{EH}$ coïncide avec une baisse de productivité. De plus, ce système exige beaucoup de temps de la part des praticiens puisque, pour respecter certaines exigences réglementaires, ces derniers doivent aussi produire des données sur l'évolution de leurs dossiers.

L'aspect le plus troublant de cette approche est le message que le choix d'un indice de productivité véhicule auprès des praticiens. Implicitement, le choix de l'indice EH implique qu'ils chercheront à maximiser les intrants, puisque la direction prête une attention particulière à cet indice. Ainsi, prendre plus de temps pour traiter un cas augmente l'indice $\mathrm{EH}$, alors qu'utiliser une approche innovatrice peut au contraire réduire le temps de traitement, et donc l'indice EH. Est-ce bien là le genre de message que la direction doit transmettre aux praticiens en période de pénurie de ressources? Ne doit-on pas au contraire retenir des indices qui valorisent l'innovation et incitent à la réduction des temps de traitement ? Seul un indice qui se veut une approximation des extrants du travail social peut atteindre cet objectif et éliminer du même coup une coûteuse et pénible duplication des systèmes de cueillette de données (sur les intrants et sur les extrants).

Parmi les approches qui cherchent à obtenir une approximation des extrants, on peut distinguer trois grandes catégories: l'évaluation de programme, le décompte des clients traités et les matrices de productivité multicritères. Le tableau 2 présente les définitions de ces approches, et le tableau 3 , les principales caractéristiques. 


\section{TABLEAU 2}

\section{Principaux types d'indices de mesure de la productivité}

\begin{tabular}{|l|l|}
\hline Type d'indice & \multicolumn{1}{|c|}{ Définition des modalités } \\
\hline $\begin{array}{l}\text { Décompte des } \\
\text { activités }\end{array}$ & $\begin{array}{l}\text { Recensement des activités des praticiens par catégories : } \\
\text { services directs aux clients, aux ressources, aux partenaires, } \\
\text { administration, etc. Les praticiens tiennentun journal d'activités. }\end{array}$ \\
\hline $\begin{array}{l}\text { Décompte des } \\
\text { cas traités }\end{array}$ & $\begin{array}{l}\text { Décompte des dossiers en cours, des dossiers fermés ou des } \\
\text { dossiers qui ont franchi une étape donnée du traitement au } \\
\text { cours d'un mois. Recensés par programme, région, unité, etc. }\end{array}$ \\
\hline Indices locaux & $\begin{array}{l}\text { Indicesdivers choisis localement par les cadres et les praticiens } \\
\text { pour donner une image globale de leur performance; par } \\
\text { exemple : dossiers fermés qui reviennent, plaintes, délais } \\
\text { excessifs, etc. }\end{array}$ \\
\hline $\begin{array}{l}\text { Évaluation de } \\
\text { programme }\end{array}$ & $\begin{array}{l}\text { Évaluation fondamentale des effets d'un programme, basée } \\
\text { surune étude de causalité entre les moyens mis en œeuvre par } \\
\text { le C.S.S. et les changements observés dans les indicateurs } \\
\text { sociaux. }\end{array}$ \\
\hline
\end{tabular}

Seule une évaluation de programmes, basée sur l'évolution des caractéristiques sociales de la population d'une région donnée, permettrait de déterminer l'amélioration du fonctionnement social de ses membres. Mais même une telle évaluation ne pourrait probablement pas établir de façon convaincante que les variations observées sont surtout attribuables aux efforts des travailleurs sociaux plutôt qu'à une foule d'autres variables non contrôlées (Elkin, 1978). De plus, le coût et le temps requis pour de telles expertises ne permettraient pas les rétroactions rapides et fréquentes dont les gestionnaires ont un pressant besoin.

Il faut donc se résoudre à élaborer des indices plus limités, mesurant plutôt l'efficience que l'efficacité, et recourir aux évaluations de programmes pour vérifier, à intervalles réguliers, que l'utilisation des indices retenus favorise bien l'atteinte des objectifs généraux de l'organisation. Comme l'efficience est une condition nécessaire mais non suffisante à l'efficacité (A.H.A., 1980), l'existence d'un contrôle renforcé de la qualité maximiserait les chances que la poursuite de l'efficience résulte en une amélioration de la productivité. 
TABLEAU 3

\section{Caractéristiques des quatre types d'indices}

\begin{tabular}{|c|c|c|c|c|}
\hline \multirow[b]{2}{*}{ Type d'indice } & \multicolumn{2}{|c|}{ Caractéristiques } & \multirow[b]{2}{*}{ Coûts } & \multirow[b]{2}{*}{$\begin{array}{l}\text { Se prête à des } \\
\text { comparaisons? }\end{array}$} \\
\hline & fiabilité/mode de validation & $\begin{array}{l}\text { Période } \\
\text { visée }\end{array}$ & & \\
\hline Décompte des activités & $\begin{array}{l}\text { peu/contrôle par les cadres } \\
\text { de première ligne }\end{array}$ & $\begin{array}{l}\text { hebdo/ } \\
\text { bimensuel }\end{array}$ & moyen & oui \\
\hline Décompte des cas traités & $\begin{array}{l}\text { fiable dans la mesure où les } \\
\text { politiques et procédures sont } \\
\text { suivies }\end{array}$ & mensuel & faible & oui \\
\hline Indices locaux & $\begin{array}{l}\text { peu/contrôle parlescadres } \\
\text { de première ligne }\end{array}$ & mensuel & faible & non \\
\hline $\begin{array}{l}\text { Évaluation de } \\
\text { programme }\end{array}$ & $\begin{array}{l}\text { fiable si fait selon les règles } \\
\text { de l'art ; question de cau- } \\
\text { salité subsiste }\end{array}$ & 2 à 5 ans & élevé & oui \\
\hline
\end{tabular}


Le décompte des dossiers traités peut s'avérer un indice intéressant, dans la mesure où des politiques éclairées d'ouverture et de fermeture de dossiers assurent une homogénéité suffisante pour faire d'un « dossier » une unité de mesure valable. Cependant, même si tel était le cas, on pourrait craindre que les variations d'un cas à l'autre dans la perception des réalités psychosociales (Coulton, Keller et Boone, 1985) soient telles que l'on additionne des quantités non comparables. La solution à ce problème passe par le regroupement des cas en catégories relativement homogènes et la pondération de ces catégories les unes par rapport aux autres. Il importe ensuite de n'effectuer des analyses que sur des ensembles suffisamment larges pour que la loi des grands nombres puisse jouer.

Finalement, le problème le plus difficile consiste à s'assurer que l'on mette constamment en parallèle des services comparables, c'està-dire que chaque dossier fermé a été classé adéquatement et a été l'objet d'un même niveau et d'une même qualité de service. Il s'agit là d'un rôle fondamental pour la direction des services professionnels du C.S.S. En plus de permettre l'élaboration d'indices de productivité uniformes et homogènes, les interventions de la D.S.P. pour définir et maintenir des catégories de services claires, uniformes et homogènes, faciliteront une dispensation ordonnée des services, permettront une meilleure coordination et un meilleur synchronisme des différents services, tout en rendant moins difficile la tâche d'assurer de la qualité. La valeur de l'indice sera également fonction de l'ardeur de la D.S.P. à assurer l'application des politiques et procédures professionnelles.

La troisième approche possible pour mesurer les extrants a été proposée par Riggs (1984). Il s'agit d'une matrice multicritères par laquelle les praticiens d'une équipe donnée choisissent un certain nombre d'indices mesurables qui leur semblent collectivement donner une représentation aussi fidèle que possible de la performance de leur équipe. Pour chaque critère, les membres de l'équipe établissent, après une étude des données historiques, une échelle et un objectif. La direction établit ensuite la pondération des critères pouvant inclure, par exemple :

- le nombre de dossiers fermés qui doivent être réouverts à l'intérieur d'une période donnée (par exemple de nouveaux signalements) ;

- le nombre de cas évalués au sujet desquels le service de prise en charge est en désaccord avec l'orientation proposée ;

- le nombre de cas urgents qui ont attendu plus d'une semaine;

- le nombre de plaintes de clients ou d'intervenants externes reliées à des erreurs de diagnostic ou à des délais d'intervention excessifs. 
En plus de permettre une plus grande participation des praticiens, et donc une meilleure acceptation des critères, cette approche peut aussi permettre la conciliation des indices globaux élaborés à partir d'un décompte du nombre de cas traités avec les indices retenus par les praticiens. Il suffit simplement d'inclure ces indices globaux dans la matrice de Riggs et de les pondérer par rapport aux autres.

Le choix de l'une ou l'autre de ces approches pour la mesure de la productivité dépendra des objectifs poursuivis (Eilon, Gold et Soesan, 1976). Tel que mentionné précédemment, les différents intervenants du C.S.S. visent souvent des objectifs différents. Le tableau 4 illustre le degré d'adéquation entre chaque catégorie d'indices et les besoins des principaux intervenants. On y note, par exemple, qu'il est relativement peu intéressant pour le M.S.S.S. - qui doit décider de l'affectation des ressources entre les programmes - de connaître l'emploi du temps des praticiens alors qu'une bonne évaluation de programme ou des données valables sur le nombre de dossiers traités lui seraient très utiles.

\section{TABLEAU 4}

\section{Utilité relative de différents types d'indices de productivité pour différents usagers}

\begin{tabular}{|c|c|c|c|c|c|}
\hline $\begin{array}{l}\text { Type Usagers } \\
\text { d'indice }\end{array}$ & Praticien & Cadre & D.S.P. & $\begin{array}{l}\text { Direction } \\
\text { générale }\end{array}$ & M.S.S.S. \\
\hline $\begin{array}{l}\text { Décompte des } \\
\text { activités }\end{array}$ & & & & & $\begin{array}{l}\text { Utilité } \\
\text { faible }\end{array}$ \\
\hline $\begin{array}{l}\text { Décompte des } \\
\text { cas traités }\end{array}$ & $\begin{array}{l}\text { Utilité } \\
\text { faible }\end{array}$ & & & & \\
\hline $\begin{array}{l}\text { Indices } \\
\text { locaux }\end{array}$ & & & & & $\begin{array}{l}\text { Utilité } \\
\text { faible }\end{array}$ \\
\hline $\begin{array}{l}\text { Évaluation de } \\
\text { programme }\end{array}$ & $\begin{array}{l}\text { Utilité } \\
\text { faible }\end{array}$ & & & & \\
\hline
\end{tabular}


On y note surtout qu'aucun indice ne satisfait pleinement les besoins de tout le monde. Rien n'empêche cependant d'en utiliser plus d'un afin de rencontrer les besoins de chacun. Si l'on choisit cette voie, il faut surtout s'assurer que l'on n'utilise pas une double tenue de livres. Il serait par exemple néfaste, à long terme, pour le M.S.S.S., d'utiliser, pour fins d'affectation des ressources, un indice que les établissements eux-mêmes n'utiliseraient pas pour leurs propres fins d'affectation interne de ressources, ou pour fins de rétroaction auprès des praticiens. Si les praticiens qui produisent les données de base nécessaires à l'élaboration de l'indice n'en reçoivent aucune rétroaction, la qualité des données de base se détériorera graduellement, au fur et à mesure qu'ils s'en désintéresseront. L'approche proposée précédemment, qui consiste à inclure les indices " officiels » dans la matrice de Riggs, semble donc, à fortiori, intéressante.

\section{Une dynamique de comparaison}

Suite à l'élaboration de nouveaux indices de productivité, la création d'une dynamique de comparaison intergroupes, dans un C.S.S., est une nécessité dictée par l'absence de normes universellement utilisables. En facilitant l'identification des différences de pratiques professionnelles qui ont un impact significatif sur la productivité, ces comparaisons constituent un mécanisme privilégié pour favoriser les échanges de bons procédés.

Puisqu'il s'agit de nouveaux indices et que les pratiques professionnelles sont très hétérogènes, les écarts entre unités administratives risquent d'être substantiels. Il est donc sage de procéder d'abord à une analyse sommaire des écarts plutôt que d'en effectuer une analyse détaillée. En procédant ainsi, on s'assure d'identifier d'abord les facteurs les plus importants, et on évite les frustrations qu'engendrerait l'application d'outils analytiques sophistiqués à des données somme toute grossières. Une approche possible consiste, après s'être assuré que les données sont bien comparables, à demander aux deux cadres dont les unités administratives présentent le contraste le plus extrême au niveau de l'indice retenu, de procéder à une comparaison systématique de leurs pratiques et de soumettre au comité de direction un rapport identifiant les facteurs explicatifs des écarts, par ordre d'importance. Le tableau 5 illustre certains des facteurs qui pourraient ressortir d'un tel exercice. L'exploration des éléments contrôlables produira une information utile à la gestion quant aux actions à poser pour améliorer la productivité. 


\section{TABLEAU 5}

\section{Principales variables pouvant expliquer les écarts interrégionaux dans les indices de productivité}

\begin{tabular}{|l|}
\hline \multicolumn{1}{|c|}{ Variables incontrôlables } \\
\hline - différences géographiques \\
- différences démographiques \\
- différences au niveau de l'environnement socio-économique et consé- \\
quemment des taux d'incidence et d'acuité des problématiques \\
- différences au niveau de la disponibilité et de la qualité des ressources \\
institutionnelles \\
- différences dans la taille des organisations \\
\hline \multicolumn{1}{c|}{ Variables contrôlables } \\
\hline - différences au niveau des pratiques professionnelles \\
- divisions du travail différentes \\
- différentes politiques quant au choix des bénéficiaires, aux priorités de \\
service et au niveau de service \\
- différences au plan des pratiques administratives \\
- différences au niveau de l'effort consenti par les praticiens ou au plan de \\
l'efficacité de ces efforts \\
- différences au niveau de la disponibilité et de la qualité des ressources \\
non-institutionnelles
\end{tabular}

Plusieurs cadres et praticiens réagissent toutefois avec méfiance à l'idée de comparaisons systématiques entre différents groupes. Certains craignent que cet exercice ne soit pas à leur avantage ; d'autres le jugent impossible puisque tous les cas sont différents. Bien qu'il n'existe pas de formule magique pour résoudre ces problèmes, il est possible de se rallier une partie des premiers en établissant des règles du jeu selon lesquelles les données ne seront utilisées pour la prise de décision que lorsqu'elles auront un niveau de fiabilité suffisant. Il importe également de rappeler qu'un mécanisme efficace de comparaison permettra d'éviter une prise de décision arbitraire. Quant aux seconds, au-delà de l'argument basé sur la loi des grands nombres, la garantie d'une consultation et d'une participation pleine et entière au projet est susceptible d'apaiser - au moins en partie - leurs craintes légitimes qu'une telle approche ne soit appliquée aveuglément. 
Si les comparaisons sont difficiles à l'intérieur d'un C.S.S., elles le sont plus encore lorsque plus d'un organisme est impliqué. Dès que l'on franchit les frontières d'une organisation, on se retrouve face à une hétérogénéité dans les définitions, les politiques et pratiques professionnelles et administratives, les systèmes d'information, la division du travail, les priorités, etc. II doit donc y avoir une étape préalable qui consiste à homogénéiser, ou tout au moins à harmoniser les différents systèmes et à vérifier leur compatibilité. Bien que cette étape puisse être longue et difficile, elle est néanmoins essentielle. De plus, il est important qu'elle n'aboutisse pas à une réduction du projet au plus petit commun dénominateur.

Finalement, les comparaisons interprogrammes posent peut-être le niveau de difficulté le plus élevé. Pourtant, l'appréciation des charges de travail respectives, dans différents projets, est un intrant essentiel à la prise de décisions éclairées quant à l'affectation des ressources entre les programmes. Toute méthodologie visant à effectuer de telles analyses comparatives devra faire usage à forte dose des jugements des experts impliqués dans les programmes concernés.

\section{Démarches parallèles}

L'amélioration du système d'information, pour assurer la disponibilité et l'exactitude des indices retenus, s'avère une contrainte au bon déroulement de tout programme d'augmentation de la productivité. Dans le domaine des services professionnels, il faut beaucoup se fier aux professionnels eux-mêmes pour la production d'une information de qualité. Par ailleurs, le temps consacré à cette tâche n'est pas du temps "productif», c'est-à-dire consacré au service à la clientèle. II importe donc d'effectuer des rétroactions fréquentes aux praticiens quant à leur performance, en plus de leur faciliter la vie (par exemple, par la saisie directe de certaines données) et de leur offrir certains bénéfices (comme la consultation en direct de certains fichiers), pour qu'ils voient l'utilité et l'importance de cette tâche.

Le virage à négocier pour améliorer le rendement ne constitue pas moins qu'un virage culturel pour un C.S.S. Depuis quelques années, l'attitude des intervenants des C.S.S. face à la productivité s'est modifiée sensiblement, sous le double effet des pressions externes et d'une dynamique interne. Mais il reste beaucoup à faire. Des modifications substantielles au niveau des systèmes de valeurs sont requises : il s'agit là d'une démarche de développement organisationnel. Le processus d'amélioration de la productivité, dans les C.S.S., doit donc être caractérisé 
par l'alternance des efforts consacrés à en améliorer la mesure et des efforts consacrés à la sensibilisation des cadres et praticiens, de même que par la dynamique de va-et-vient du haut de la pyramide organisationnelle jusqu'au bas, et vice-versa.

Le renforcement de la gestion des opérations est probablement le changement majeur qui doit survenir dans les C.S.S. Une dynamique de comparaisons interrégions et intertemporelles doit prendre racine. Cette dynamique doit être assortie d'un esprit de recherche et d'une motivation à améliorer la production. Grâce au meilleur contrôle de gestion qu'ils permettent, les indices de productivité devraient favoriser une décentralisation réelle de la gestion. Les cadres de première ligne et les praticiens, qui sont les mieux placés pour améliorer la productivité, disposeront ainsi d'une plus grande latitude pour ce faire, pourvu qu'ils acceptent de rendre des comptes. Loin de constituer une menace, la mise en place d'indices de productivité constitue une opportunité pour les intervenants sociaux de démontrer l'importance de leur contribution (A.H.A. 1980).

\section{Quelques expériences québécoises}

Les C.S.S. du Québec ne sont pas sans ressentir les nombreuses pressions qui s'exercent sur eux pour améliorer leur productivité; l'absence d'une mesure fiable les place toutefois devant la double impossibilité de la démontrer, le cas échéant, ou, dans le cas contraire, d'identifier les programmes, régions, unités administratives ou praticiens qui pèchent à cet égard. Face à la nécessité d'agir et à la complexité du problème, ils ne restent pas inactifs cependant. Chacun, à sa façon, cherche à améliorer le rendement et plusieurs reconnaissent que pouvoir le mesurer en est une condition préalable. Toutefois, tous ne choisissent pas la même voie. Nous décrirons, dans les lignes qui suivent, quatre expériences, récentes ou présentement en cours, de mise en place d'indices opérationnels. Nous avons été étroitement associé aux deux premières expériences présentées.

\section{C.S.S. A : comparaison inter-équipes de différents indices opérationnels à la prise en charge}

Il y a deux ans, le C.S.S. A a amorcé une dynamique de comparaison de certains indices opérationnels entre les équipes responsables de la prise en charge (jeunesse, adultes, handicapés physiques et mentaux) dans les divers territoires qu'il dessert. 
Le premier indice retenu fut le caseload, c'est-à-dire le nombre de dossiers (ou cas) dont un praticien a la responsabilité, à un moment donné. Le C.S.S. A produisait déjà mensuellement un rapport à ce sujet, mais qui n'était toutefois pas l'objet d'un suivi systématique au comité de direction.

L'utilisation du caseload présente deux avantages importants : tous les travailleurs sociaux sont familiers avec cette notion, et des études ont été menées aux États-Unis pour établir des normes approximatives.

Des études ponctuelles furent donc préparées, au C.S.S. A, comparant les charges moyennes par unité administrative, afin de permettre des discussions au niveau du comité de direction et des comités de gestion de chaque territoire. Ces comparaisons révélèrent des écarts importants, allant presque du simple au double. Les directeurs régionaux, qui ne pouvaient s'expliquer de tels écarts, résolurent de se pencher sur la question. La D.S.P. fut associée à cette démarche laquelle révéla des pratiques fort diverses au niveau de l'ouverture et de la fermeture des dossiers: certains, qui auraient dû être fermés depuis longtemps, étaient toujours ouverts alors que d'autres n'étaient jamais ouverts. Comme les praticiens n'avaient jamais vraiment reçu de réactions de la direction quant à cet indice, ils s'en étaient désintéressés, ce qui avait permis que de telles situations perdurent.

Pour pallier à cet état de chose, la D.S.P. instaura, à l'échelle de l'établissement, une politique de révision systématique, imposant à chaque cadre de lui faire rapport mensuellement sur l'état d'un certain nombre de dossiers. Malgré cette uniformisation des pratiques, des écarts substantiels persistèrent entre les régions.

L'attention que portait la direction à la productivité, telle que mesurée par le caseload, pouvait toutefois susciter des comportements "défensifs " chez certains cadres et praticiens. En effet, constatant que la fermeture d'un dossier amenait une baisse de l'indice (caseload), certains praticiens pouvaient être tentés de garder des dossiers très peu actifs qui auraient dû être fermés. De plus, la tentation était omniprésente de ne pas fermer leurs cas légers, de crainte de les voir remplacés par des cas beaucoup plus lourds.

Certains problèmes furent également constatés au niveau de la liste d'attente quant aux demandes de services ne pouvant être remplies faute de praticien disponible. Cette liste est normalement récensée au niveau des cadres de première ligne. Certains cadres (une minorité) ont toutefois trouvé doublement avantageux d'éliminer leur liste d'attente, en attribuant, sur réception, toutes les demandes à leurs praticiens plutôt que d'attendre la disponibilité de l'un d'eux; on augmente ainsi la charge de cas des praticiens et on évite les questions embarrassantes 
suscitées par une trop longue liste d'attente. Malheureusement, cette pratique n'augmente pas la production; elle ne fait que déplacer la file d'attente du cadre vers les praticiens, la rendant du même coup invisible pour la direction générale, ce qui équivaut à une abdication du cadre face à son rôle fondamental d'établir les priorités de service.

À cause de ces problèmes, le caseload ne pouvait être retenu comme seul indice de productivité. Un nouvel indice était donc requis, qui ne pénaliserait pas les praticiens qui ferment leurs cas à temps, qui mettrait au contraire l'accent sur la production, c'est-à-dire la nécessité de fermer les dossiers, et qui n'inciterait pas les cadres à attribuer prématurément les cas en attente.

Le second indice évalué fut le taux de roulement des cas. Il s'agit là d'une mesure dynamique car elle reflète le niveau d'activité pendant une période de temps plutôt que de mettre l'accent sur la situation à un moment donné (comme le fait le caseload). Cet indice fut défini de la façon suivante :

52 semaines

$$
\text { Taux de roulement }=\frac{}{\text { durée moyenne de service (en semaines) }}
$$

Tel que souhaité, cet indice mettait nettement l'accent sur la nécessité de fermer les dossiers et corrigeait ainsi les lacunes décrites précédemment. Cet indice était toutefois incomplet puisqu'il ne tenait compte que du roulement des cas sans prendre en considération le nombre absolu de cas traités. De plus, cette mesure n'était pas familière aux travailleurs sociaux et il n'existait aucune norme à laquelle le C.S.S. pouvait se référer. Pour pallier à la première faiblesse, il fut suggéré d'utiliser simultanément les deux indices (caseload et taux de roulement). Quant à la seconde, même s'il n'existait pas de norme de taux de roulement, il était tout de même possible de comparer les régions entre elles, et c'est cette voie qui fut retenue. Par la suite, un indice unique incorporant les deux précédents - le nombre de dossiers fermés par période - fut ajouté.

Chacune des étapes de ce projet a nécessité beaucoup de temps et d'énergie : après avoir choisi un indice, il a fallu définir un mode de calcul précis, modifier les systèmes informatiques, modifier la saisie des données de base, informer et former les participants, cueillir et " nettoyer " les données, etc.

\section{Comparaisons inter-organismes à l'Association des C.S.S.}

Cinq C.S.S. de diverses tailles se sont portés volontaires pour participer à un projet pilote coordonné par l'auteur, sous l'égide de 
l'Association des C.S.S. Ce projet consistait à comparer, selon les modalités définies ci-haut, la productivité des participants au niveau du volet accueil/évaluation/orientation du programme de protection de la jeunesse. Outre les difficultés considérables susmentionnées, l'uniformisation des définitions et l'harmonisation des systèmes informatiques, un problème de taille s'est posé dès le début: certains types de problématiques, nécessitant des interventions beaucoup plus longues, sont plus fréquents dans certains C.S.S. que dans d'autres. II n'est donc pas possible d'additionner sans discrimination tous les cas traités, indépendamment des problématiques. L'élaboration d'une taxonomie valable n'est pas chose facile dans les services sociaux (Coulton, 1979). Celle-ci doit permettre de regrouper, pour fins de calculs, les cas nécessitant des durées d'intervention semblables. Il faut ensuite pondérer ces catégories d'intervention les unes par rapport aux autres, en termes de leurs durées relatives.

Deux avenues s'offrent à nous pour effectuer cette pondération :

- étude de temps, à l'aide d'un journal d'activités ou de techniques de mesure du travail (chronomètre, échantillonnage du travail, etc.) ;

- recours à l'expertise des professionnels pour effectuer une estimation subjective des poids, à l'aide de la méthode des comparaisons par paires de Thrustone (1927) ou d'une approche delphienne (Linstone et Turoff, 1975).

La première avenue nous conduit presque à coup sûr à une résistance farouche des professionnels, et donc à l'échec du projet, puisque leur collaboration est essentielle. La seconde, par ailleurs, permet d'utiliser une banque de connaissances des plus riches, tout en associant dès le départ les professionnels à la démarche. Toutefois, bien que les deux méthodes mentionnées aient une valeur scientifique démontrée, il n'est pas impossible que l'on se heurte à une certaine méfiance de la part des organismes de financement par rapport à ces données. Face à ce dilemme, les participants ont opté pour la seconde avenue, tout en prenant tous les moyens possibles pour assurer un maximum de rigueur à l'étude et pour valider les données obtenues.

\section{C.S.S. B : caseload pondéré par un indice de lourdeur des cas}

Lorsqu'on veut utiliser le caseload pour comparer la productivité de différents praticiens au cours d'une période de temps limitée, le nombre de dossiers traités n'est généralement pas suffisant pour que les 
moyennes soient comparables. En effet, certains praticiens auront des dossiers plus lourds que d'autres, en moyenne. Si l'on veut utiliser l'indice de productivité pour favoriser une plus grande uniformité dans les charges de travail, c'est-à-dire l'utiliser comme guide pour l'attribution des nouveaux cas, il faut donc pondérer chaque cas en y attachant un facteur reflétant sa "lourdeur relative". Puisqu'un tel facteur doit être établi pour chaque cas, il y a plusieurs choix importants à faire quant au mode de fonctionnement d'un tel système :

- Comment ou sur quelle base les facteurs de pondération sont-ils déterminés? Le C.S.S. B a établi que la pondération serait directement proportionnelle au nombre d'interventions professionnelles requises dans un dossier au cours d'une année. Il considère en effet, qu'en moyenne, une " intervention " constitue une " unité mesurée du travail " valable pour fins de comparaison des charges de travail.

- Qui doit établir la pondération? Le C.S.S. B a confié cette responsabilité à chaque cadre de premier niveau. Une variante intéressante consisterait à effectuer cette évaluation conjointement avec certains praticiens, de manière à assurer la confrontation d'une variété de points de vue, et à assurer une meilleure acceptation grâce à une plus forte participation.

- Quelle est la charge de travail normale ? L'utilisation des pondérations amène l'établissement d'un indice quantitatif (caseload pondéré) pour chaque praticien. II devient alors inévitable de déterminer quantitativement ce qui constitue une charge «normale ». Pour ce faire, le C.S.S. B s'est basé sur la moyenne observée pour établir une "zone normale", définie par une charge minimale et une charge maximale. En procédant ainsi, on laisse une certaine marge de manœuvre aux cadres, qui doivent aussi considérer d'autres facteurs pour l'attribution des cas tels les expertises et intérêts particuliers des praticiens, les autres tâches confiées à chacun, etc. Très large au niveau de la charge mensuelle d'un praticien, la zone normale sera évidemment beaucoup plus étroite à plus long terme, c'est-à-dire en regard d'une charge annuelle.

- Quels contrôles doit-on exercer sur les pondérations ? Le C.S.S. $B$, qui en est à la phase d'expérimentation, n'a pas encore établi de mécanismes de contrôle pour s'assurer d'une certaine uniformité entre unités administratives, dans l'établissement des pondérations. II est en effet probable que certains cadres aient tendance à estimer plus généreusement que d'autres le nombre d'interventions requises. À moyen terme, il serait donc utile de 
mettre sur pied des mécanismes qui assurent une rétroaction, pour que chaque cadre sache où il se situe par rapport aux autres et puisse graduellement ajuster son tir.

Le C.S.S. B doit décider prochainement si l'expérience s'avère suffisamment concluante pour passer à la phase d'implantation de ce système dans tous les programmes.

\section{C.S.S. C : système d'information sur les activités des praticiens}

Le C.S.S. C s'apprête à lancer, à titre de projet pilote, une fiche d'inscription des activités, qui permettra d'estimer les temps unitaires moyens des «principales activités de production » des praticiens. Chaque praticien serait alors tenu de remplir quotidiennement une fiche faisant état pour chaque dossier des interventions principales faites durant la journée, par secteur d'activités (service direct à un client, administration, etc.), par étape (réception, évaluation, etc.) et sous-étape (planification, réunion, déplacement, etc.)

La mise sur pied d'un tel projet est un exercice à grand déploiement pour un C.S.S. et demande une planification de longue main. Le choix du niveau de détails dans la décomposition du travail, la minimisation du temps requis par le praticien pour compléter la fiche, la communication du projet à toutes les parties concernées, l'uniformisation des méthodes de cueillette de données, la modification des systèmes informatiques, et l'intégration des données ainsi produites dans le processus de gestion du C.S.S., sont toutes des tâches délicates et complexes qui doivent être étroitement coordonnées.

\section{Conclusion}

Les professionnels et les bureaucraties des services préfèrent généralement, lorsque c'est possible, s'en tenir au qualitatif, qui est somme toute le propre d'un service professionnel, et se rebiffent face aux nombreuses difficultés et aux importantes contraintes rencontrées lorsque l'on cherche à obtenir des indices quantitatifs (Mintzberg, 1979). La conjoncture économique québécoise, alliée à un plus grand souci d'imputabilité dans le réseau des affaires sociales, amènent toutefois les C.S.S. à réviser leur position face au dilemme quantité/qualité dans la gestion des services sociaux. 
Malgré ces difficultés, l'élaboration d'indices de productivité s'avère utile pour la gestion et pour les professionnels. Le processus de choix d'un indice et du suivi systématique de la productivité amène les cadres et les intervenants à s'interroger sur la conformité de leur pratique avec les politiques et les priorités de l'établissement. II s'avère de plus un stimulant important aux échanges de bons procédés, qui sont une source privilégiée d'amélioration de la productivité dans les services professionnels. De plus, il réduit sensiblement l'arbitraire au niveau de l'affectation des ressources.

II ne faut toutefois pas s'engager aveuglément dans cette voie et prétendre que toute la complexité des services sociaux peut se résumer en quelques chiffres. Si des indices de gestion sont sans contredit nécessaires, leur portée et leurs limites doivent être définies avec précision, et ils ne doivent constituer que l'un des intrants parmi d'autres à la prise de décision, telles les évaluations de programmes, les études portant sur la qualité des services, les opinions des experts.

\section{Note}

1 Formule simplifiée. Un facteur de normalisation est également inclus dans la formule complète.

\section{Références bibliographiques}

American Hospital Association (A.H.A.), "Cost Accountability for Hospital Social Work ", Chicago, Society for Hospital Social Work Directors, no 1330, 1980.

BRItNeY, R.R., D. JOHNSTON, J.M. LEGENTIL et J. WALSH, «Planning for Productivity Gains Within the Firm: a Management Perspective", School of Business Administration, University of Western Ontario, 1982. ("Working Paper Series ", 82-38.)

Coulton, C.J., "Social Work Quality Assurance Programs : A Comparative Analysis ", Washington, National Association of Social Workers, 1979.

Coulton, C.J., S.M. Keller et C.E. BONE, "Predicting Social Workers' expenditure of time with hospital patients", Health and Social Work, vol. 10, no 1, 1985, pp. 35-44.

EILon, S., B. Gold et J. Soesan, Applied Productivity Analysis for Industry, New York, Pergamon Press, 1976. 
ElkiN, E., "Stepping Stone to Preserving Needed Human Service Programs", Management Focus, vol. 25, n 6, 1978.

KENDRICK, J.W., "Improving Company Productivity ", American Productivity Center, Baltimore, The John Hopkins University Press, 1984.

Linstone, H.A. et M. Turoff (éds), The Delphi Method: Technique and Application, Reading (Mass.), Addison-Wesley, 1975.

MintzBerG, H., "The structuring of Organizations", Englewood Cliffs (N.J.), Prentice Hall, 1979.

RIGGs, J.L., "The Objectives Matrix for Productivity Measurement ", Operations Management Review, vol. 2, no 4, 1984.

SIME, K.C. et J.M. FARBER, "A Comprehensive Computerized Management Information System for Social Workers ", Toronto, Joint National Conference of the Canadian Association of Social Work Administrators in Health Facilities and the Society for Hospital Social Work Directors, 1982.

Thurstone, L.L., "A Law of Comparative Judgment ", Psychology Review, vol. 34, $1927: 273-286$. 